\title{
A zipcode unzipped
}

\author{
Michael Doyle and Michael A. Kiebler ${ }^{1}$ \\ Center for Brain Research, Medical University of Vienna, 1090 Vienna, Austria
}

RNA-binding proteins (RBPs) exert many roles in the post-transcriptional regulation of gene expression in eukaryotic cells. However, our understanding of how they recognize their target RNAs in vivo remains limited. In the January 1, 2012, issue of Genes \& Development, Patel and colleagues (p. 43-53) provide detailed mechanistic insights into how one of the best-studied RBPs, zipcode-binding protein 1 (ZBP1), recognizes a bipartite RNA sequence element within the $\beta$-actin mRNA.

From the cradle (transcription) to the grave (RNA decay), an mRNA will interact with many different RNA-binding proteins (RBPs). These RBPs play key roles in the posttranscriptional regulation of gene expression (Burd and Dreyfuss 1994), such as RNA quality control, nuclear export, and cytoplasmic localization, as well as eventual translation and decay. To date, several distinct types of RNA-binding motifs have been identified within different types of RBPs (Stefl et al. 2005; Lunde et al. 2007). These motifs allow a given RBP to interact with its targets in a specific manner in the cell. Often, these RBPs bind to their transcripts along with other protein factors to form a larger ribonucleoprotein (RNP) complex. RNPs represent true molecular machines that exert multiple functions and determine the fate of their associated transcripts. The importance of the RBP/target mRNA interaction is illustrated by the fact that several genetic disorders are caused by the aberrant expression or malfunction of RBPs (Burd and Dreyfuss 1994; Lukong et al. 2008)

The initial RBP/target mRNA interaction occurs cotranscriptionally, as the transcript grows in length. At this stage, key interacting RBPs include splicing factors and the exon-junction complex (EJC) as well as RNA chaperones, which contribute to the folding of the RNA into a secondary or even tertiary structure (Lorsch 2002). Once an RNA is folded, other RBPs will bind by recognizing either specific primary sequences, a particular structure, or possibly a combination of both (Stefl et al. 2005). We are now beginning to understand in detail how RBPs specifi-

[Keywords: ZBP1; RNA-binding protein; KH domain; RNA localization] ${ }^{1}$ Corresponding author.

E-mail Michael.Kiebler@meduniwien.ac.at.

Article is online at http://www.genesdev.org/cgi/doi/10.1101/gad.184945.111. Freely available online through the Genes \& Development Open Access option. cally recognize their RNAs. Often, individual RNA-binding domains (RBDs) only recognize a certain sequence with low affinity. Since any given RBP often contains several different RBDs, it is hypothesized that the domains cooperate to achieve a higher specificity of RBP interaction and biological activity. Such cooperativity may take place either intra- or intermolecularly. Here, we present examples for both types of interaction that can explain two important features of RBP/RNA function. First, RBPs are able to bind their biological targets with high affinity despite the fact that their individual domains exert only low specificity. Second, cooperativity of RBDs within a given RBP may also explain how an RBP can selectively distinguish between different transcripts within the cell.

It is in this context that two recent studies by Singer and coworkers (Chao et al. 2010; Patel et al. 2012) provide another attractive mechanism as to how an RBP might interact with its cargo RNA. They studied the zipcodebinding protein (ZBP1/IMP1/IgfIIBP1), which is involved in the regulation of $\beta$-actin mRNA, the first transcript shown to localize within fibroblasts and neurons. The investigators propose that ZBP1 interacts with an unfolded or loosely folded RNA and that this interaction forces the RNA to adopt a secondary structure by wrapping around ZBP1-what they termed the looping model (see Fig. 1).

\section{A crash course on ZBP1}

ZBP1-a member of a highly conserved family of RBPsplays important roles in the localization, stability, and translational control of cellular mRNAs (Yisraeli 2005). The protein contains six canonical RBDs: two RNA recognition motifs (RRMs) and four hnRNP K homology (KH) domains (Ross et al. 1997). Previous work has shown that the KH3 and KH4 (Chao et al. 2010) domains preferentially mediate RNA recognition of a 54-nucleotide (nt)-long degenerate RNA zipcode sequence. This sequence motif was originally found in $\beta$-actin mRNA (Kislauskis et al. 1994). ZBP1 KH34 domains bind a 28-nt-long sequence within the zipcode (Farina et al. 2003), and multimerization does not appear to be required for stable RNA recognition (Chao et al. 2010). Structural data of IMP1, the human homolog of ZBP1, showed that the KH34 domains form an intramolecular pseudodimer. This arrangement of the RNA-binding surfaces of $\mathrm{KH} 3$ and $\mathrm{KH} 4$ at opposing ends of ZBP1 led the investigators to propose an attractive model whereby the two RNA recognition elements (REs) within the zipcode have to be separated by a spacer. 


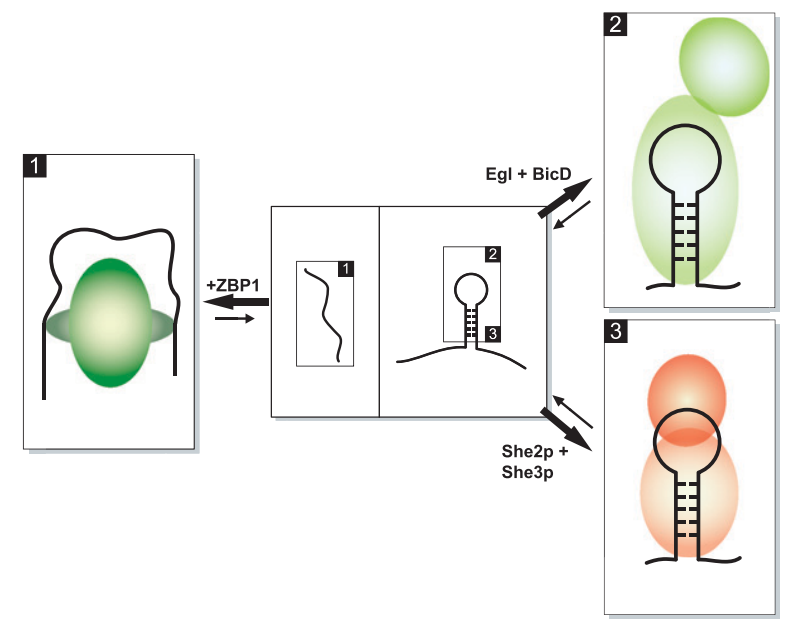

Figure 1. Intra- and intermolecular cooperativity models of RBPs and their substrate recognition. Shown in the center are unstructured (left center panel) and structured (right center panel) RNAs. (Box 1) In the case of $\mathrm{ZBP} 1$, it recognizes an RNA zipcode in the $\beta$-actin transcript and binds the RNA via two KH domains forcing a conformational change in the RNA. (Box 2) For Egl and BicD, the RBP Egl recognizes the stem-loop of K10 RNA. Subsequent binding of the dynein adaptor BicD to Egl then increases its affinity for RNA. (Box 3) For She2p and She3p, both proteins cooperate together for ASH1 RNA binding. Proteins are depicted as colored spheres, and RNA is shown as black lines.

This allows the RNA to loop around ZBP1 and adopt an anti-parallel orientation. It is worth noting that when this spacing was shortened by $\geq 6 \mathrm{nt}$, RNA binding was severely abrogated.

\section{A detailed look at RNA binding by ZBP1}

In their new study, Patel et al. (2012) extend previous work from the Singer laboratory by focusing on both the $5^{\prime}$ and $3^{\prime}$ ends of the $\beta$-actin zipcode. They performed polyacrylamide gel electrophoretic mobility shift assay (EMSA) experiments to compare ZBP1 binding to wild-type and mutant RNA sequences. Previous work had shown that single $\mathrm{KH}$ domains often recognize short, $\sim$ 4-nt-long stretches within their target RNAs. Although a highly conserved 5' RE (5RE) was identified (CGGAC), there was no strong conservation in the $3^{\prime} \mathrm{RE}$ (3RE; C/A-CA$\mathrm{C} / \mathrm{U})$. Owing to the abundance of cytosines in the $3 \mathrm{RE}$, in vitro SELEX experiments (rather than single point mutants) were used to define the RNA/protein interaction. The orientation of KH34 with respect to the RNA was then determined using nuclear magnetic resonance (NMR) spectroscopy of KH34 in complex with either the 5RE or 3RE. These experiments predict that KH4 binds to the $5 \mathrm{RE}$, whereas $\mathrm{KH} 3$ recognizes the $3 \mathrm{RE}$. The investigators also swapped the positions of the $5 \mathrm{RE}$ and $3 \mathrm{RE}$ within the zipcode. While this new arrangement would be expected to alter the position of the RNA with respect to ZBP1 such that the spacer region within the RNA is now opposite to the KH34 linker, it did not affect RNA binding. This suggested that the protein linker does not make direct contact with the looped RNA.

\section{A more general consensus for ZBP1 target mRNAs}

The characterization of a defined spacer-containing, bipartite RE consensus sequence allowed the investigators to predict target mRNAs for ZBP1. Both the human and mouse transcriptomes were analyzed, and 114 evolutionarily conserved targets were identified. Although previous studies reported additional targets for ZBP1 (see Chao et al. 2010 and references therein; Hafner et al. 2010), this novel computational approach yielded an extensive list of potential new candidate RNAs for ZBP1. The importance of the new candidates was underlined by gene ontology analysis, which identified categories with developmental and morphological functions, and a specific emphasis on neurological diseases and cancer. In fact, the prediction that ZBP1 has important functions during development correlates with the observation that it is primarily expressed during early embryogenesis. The investigators next experimentally verified a subset of potential new candidates by performing ZBP1 immunoprecipitation experiments in mouse embryonic brain extracts, isolating the associated RNAs, and then analyzing their abundance by quantitative PCR (qPCR). Rewardingly, 17 of the 24 tested transcripts were indeed enriched by more than fourfold in ZBP1 immunoprecipitates. These findings support the investigators' multipronged approach to include both spatial information and sequence specificity in the identification of mRNA targets for ZBP1.

\section{ZBP1-dependent dendritic targeting of spinophilin mRNA?}

Interestingly, one of the identified mRNAs, which codes for spinophilin, had previously been suggested to localize to dendrites of hippocampal neurons (Zhong et al. 2006). When both copies of the ZBP1-binding sites in the spinophilin sequence were deleted from a reporter construct, there was a marked decrease in dendritic localization. Furthermore, the investigators took advantage of ZBP1 knockout mice (Z Katz and RH Singer, in prep.) and investigated whether spinophilin mRNA localization is indeed affected in dendrites from ZBP1-deficient mouse neurons. Whereas the number of spinophilin mRNA puncta in dendrites was diminished twofold in knockout animals compared with wild type, its localization to distal dendrites was not significantly affected. The investigators therefore concluded that, most likely, ZBP1 works with other RBPs to target spinophilin mRNA to dendrites. Consequently, an interesting question for future studies is whether ZBP1 contributes to dendritic trafficking or whether it affects RNA stability and/or translational control.

\section{A new form of RNA recognition for an RBP}

Patel et al. (2012) identified a novel mechanism of cargo RNA recognition by an RBP-in this case, $\beta$-actin mRNA by ZBP1. As outlined above, many RBPs often contain multiple copies of a single type of $\mathrm{RBD}$, such as the $\mathrm{KH}$ 
domain. Interestingly, it was previously reported that $\mathrm{KH}$ domains bind their targets either independently or cooperatively (Paziewska et al. 2004). However, the current study extends our knowledge by providing detailed insight into how two $\mathrm{KH}$ domains cooperate in RNA recognition. As outlined above, cooperativity can occur either within an RBP (intramolecular) or between several RBPs (intermolecular). Examples for intramolecular cooperativity are distinct dsRBDs in various proteins, including the Staufen proteins (Lunde et al. 2007), or the RNA-editing enzymes ADAR1 and ADAR2 (Doyle and Jantsch 2003; Macbeth et al. 2004). In addition, the two RRMs within the Drosophila sex determination protein Sex Lethal work cooperatively to determine alternative splicing of its own transcript as well as transformer (Handa et al. 1999). For intermolecular cooperativity, there are (at least) two prominent examples in which two distinct proteins act together to recognize RNA structural elements. The first example is from Drosophila, where two proteins cooperate to yield high-affinity recognition of a highly structured RNA stemloop within the K10 RNA (Dienstbier et al. 2009). Here, the RBP Egalitarian (Egl) recognizes localization elements in the K10 RNA through an unidentified RBD (see Fig. 1, inset 2). Binding of the dynein cofactor Bicaudal-D (BicD) to Egl then increases the affinity of Egl for the RNA. In the second example, two yeast RBPs, She2p and She3p, both recognize structured REs within ASH1 mRNA (Müller et al. 2011). Acting synergistically, they dramatically increase specificity and affinity in binding to the structured ASH1 RNA (see Fig. 1, inset 3; Müller et al. 2011). However, much less is known about how the two She proteins bind their RNA (Niessing et al. 2004; Müller et al. 2011).

\section{How does RNA recognition work for an unfolded RNA?}

In contrast to the intermolecular cooperativity examples described above, where two distinct proteins recognize structured RNA elements, the two tandem KH domains of ZBP1 recognize short, primary sequences within a bipartite sequence of an unfolded RNA (Patel et al. 2012). Whereas the initial contact of one $\mathrm{KH}$ domain is of low affinity, the cooperativity between both RBDs results in a high-affinity RNA recognition. The identification of an essential spacer region (Chao et al. 2010) not only imposes further constraints to the RNA, but also enables it to change its structural conformation. Whereas the unbound RNA is unfolded or flexible, binding by the two $\mathrm{KH}$ domains in ZBP1 forces the RNA to wrap around the protein (Fig. 1, inset 1 ). This induces an $\sim 180^{\circ}$ change in the orientation of the RNA, allowing it to be in direct contact with both $\mathrm{KH} 3$ and $\mathrm{KH} 4$ that are positioned on the opposing sides of ZBP1 (Chao et al. 2010).

Taken together, this novel mode of RNA recognition by an RBP has several important implications. First, the consensus site for a single RBD does not reflect how fulllength RBPs bind to and regulate their mRNA targets in vivo. Consequently, the observed cooperativity of the $\mathrm{KH}$ domains, together with the novel spatial constraints of the RNA, helps to better explain the highly specific affinity recognition. Second, as pointed out by the investigators of this new study, knowledge of the structural arrangement of several RBDs within an RBP will allow us to improve our understanding of how a given RBP discriminates between different transcripts. Recently, Sattler and colleagues (Mackereth et al. 2011) identified two distinct conformations of RRM12 in the splicing factor U2AF64. In this case, splicing is modulated by the presence or absence of a strong polypyrimidine tract in the substrate (Mackereth et al. 2011).

Ultimately, the hypothesis-driven approach by Patel et al. (2012) will pave the way for the unraveling of complex RNA-binding specificities in the future. Their data add another layer to the "RNA signature" concept that was recently proposed (Doyle and Kiebler 2011). Here, an mRNA can be viewed as having a unique signature of multiple regulatory elements at both the primary sequence and structural levels that are recognized by certain trans-acting factors or RBPs. In this RNA-centric view, it is the RNA that recruits various RBPs, which are then responsible for the downstream functions; e.g., mRNA stability, mRNA localization, translational control, and local protein synthesis, among many others. Therefore, the latest study by Patel et al. (2012) adds another dynamic level of complexity to the RNA signature, as they demonstrate that an RBP can modulate the RNA by forcing a conformational change. It is the RBP-in their case, ZBP1- that is the key player acting on the RNA, and not vice versa. Therefore, it will be interesting to see in the future, for other RBPs and RNAs, who is the driver and who is the passenger in this dynamic relationship.

\section{Acknowledgments}

We thank Dr. Dierk Niessing, as well as members of the Kiebler laboratory, for thoughtful comments, and Sebastian Butter for assistance with figure preparation. Our work is supported by grants from the FWF (SFB F4314-B09 "RNA regulation of the transcriptome" and P-20583-B12), the HFSP, and the ESF RNA quality control (FWF I127-B12).

\section{References}

Burd CG, Dreyfuss G. 1994. Conserved structures and diversity of functions of RNA-binding proteins. Science 265: 615-621.

Chao JA, Patskovsky Y, Patel V, Levy M, Almo SC, Singer RH. 2010. ZBP1 recognition of $\beta$-actin zipcode induces RNA looping. Genes Dev 24: 148-158.

Dienstbier M, Boehl F, Li X, Bullock SL. 2009. Egalitarian is a selective RNA-binding protein linking mRNA localization signals to the dynein motor. Genes Dev 23: 1546-1558.

Doyle M, Jantsch MF. 2003. Distinct in vivo roles for doublestranded RNA-binding domains of the Xenopus RNA-editing enzyme ADAR1 in chromosomal targeting. I Cell Biol 161: 309-319.

Doyle M, Kiebler MA. 2011. Mechanisms of dendritic mRNA transport and its role in synaptic tagging. EMBO J 30: 35403552.

Farina KL, Huttelmaier S, Musunuru K, Darnell R, Singer RH. 2003. Two ZBP1 KH domains facilitate $\beta$-actin mRNA localization, granule formation, and cytoskeletal attachment. J Cell Biol 160: 77-87. 
Hafner M, Landthaler M, Burger L, Khorshid M, Hausser J, Berninger P, Rothballer A, Ascano M Jr, Jungkamp AC, Munschauer M, et al. 2010. Transcriptome-wide identification of RNA-binding protein and microRNA target sites by PAR-CLIP. Cell 141: 129-141.

Handa N, Nureki O, Kurimoto K, Kim I, Sakamoto H, Shimura Y, Muto Y, Yokoyama S. 1999. Structural basis for recognition of the tra mRNA precursor by the Sex-lethal protein. Nature 398: 579-585.

Kislauskis EH, Zhu X, Singer RH. 1994. Sequences responsible for intracellular localization of $\beta$-actin messenger RNA also affect cell phenotype. J Cell Biol 127: 441-451.

Lorsch JR. 2002. RNA chaperones exist and DEAD box proteins get a life. Cell 109: 797-800.

Lukong KE, Chang KW, Khandjian EW, Richard S. 2008. RNAbinding proteins in human genetic disease. Trends Genet 24: 416-425.

Lunde BM, Moore C, Varani G. 2007. RNA-binding proteins: Modular design for efficient function. Nat Rev Mol Cell Biol 8: 479-490.

Macbeth MR, Lingam AT, Bass BL. 2004. Evidence for autoinhibition by the $\mathrm{N}$ terminus of hADAR2 and activation by dsRNA binding. RNA 10: 1563-1571.

Mackereth CD, Madl T, Bonnal S, Simon B, Zanier K, Gasch A, Rybin V, Valcárcel J, Sattler M. 2011. Multi-domain conformational selection underlies pre-mRNA splicing regulation by U2AF. Nature 475: 408-411.

Müller M, Heym RG, Mayer A, Kramer K, Schmid M, Cramer P, Urlaub H, Jansen RP, Niessing D. 2011. A cytoplasmic complex mediates specific mRNA recognition and localization in yeast. PLOS Biol 9: e1000611. doi: 10.1371/journal. pbio.1000611.

Niessing D, Hüttelmaier S, Zenklusen D, Singer RH, Burley SK. 2004. She2p is a novel RNA-binding protein with a basic helical hairpin motif. Cell 119: 491-502.

Patel VL, Mitra S, Harris R, Buxbaum AR, Lionnet T, Benowitz M, Girvin M, Levy M, Almo SC, Singer RH, et al. 2012. Spatial arrangement of an RNA zipcode identifies mRNAs under post-transcriptional control. Genes Dev 26: 43-53.

Paziewska A, Wyrwicz LS, Bujnicki JM, Bomsztyk K, Ostrowski J. 2004. Cooperative binding of the hnRNP K three $\mathrm{KH}$ domains to mRNA targets. FEBS Lett 577: 134-140.

Ross AF, Oleynikov Y, Kislauskis EH, Taneja KL, Singer RH. 1997. Characterization of a $\beta$-actin mRNA zipcode-binding protein. Mol Cell Biol 17: 2158-2165.

Stefl R, Skrisovska L, Allain FH. 2005. RNA sequence- and shape-dependent recognition by proteins in the ribonucleoprotein particle. EMBO Rep 6: 33-38.

Yisraeli JK. 2005. VICKZ proteins: A multi-talented family of regulatory RNA-binding proteins. Biol Cell 97: 87-96.

Zhong J, Zhang T, Bloch LM. 2006. Dendritic mRNAs encode diversified functionalities in hippocampal pyramidal neurons. BMC Neurosci 7: 17. doi: 10.1186/1471-2202-7-17. 


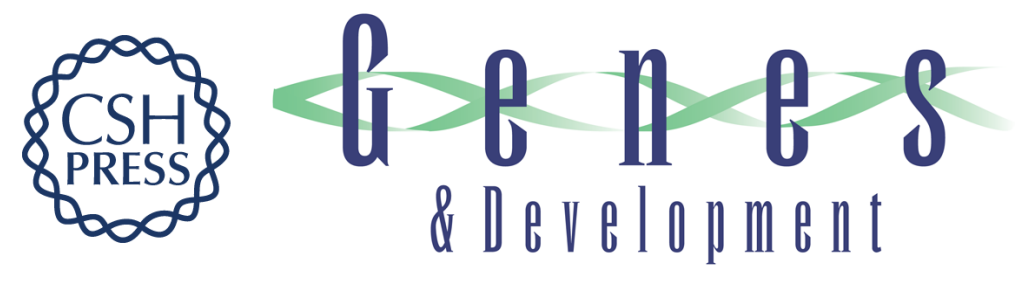

\section{A zipcode unzipped}

Michael Doyle and Michael A. Kiebler

Genes Dev. 2012, 26:

Access the most recent version at doi:10.1101/gad.184945.111
Related Content Spatial arrangement of an RNA zipcode identifies mRNAs under post-transcriptional control
Vivek L. Patel, Somdeb Mitra, Richard Harris, et al.
Genes Dev. January, 2012 26: 43-53

References This article cites 22 articles, 10 of which can be accessed free at: http://genesdev.cshlp.org/content/26/2/110.full.html\#ref-list-1

Articles cited in:

http://genesdev.cshlp.org/content/26/2/110.full.html\#related-urls

License Freely available online through the Genes \& Development Open Access option.

Email Alerting Receive free email alerts when new articles cite this article - sign up in the box at the top Service right corner of the article or click here.

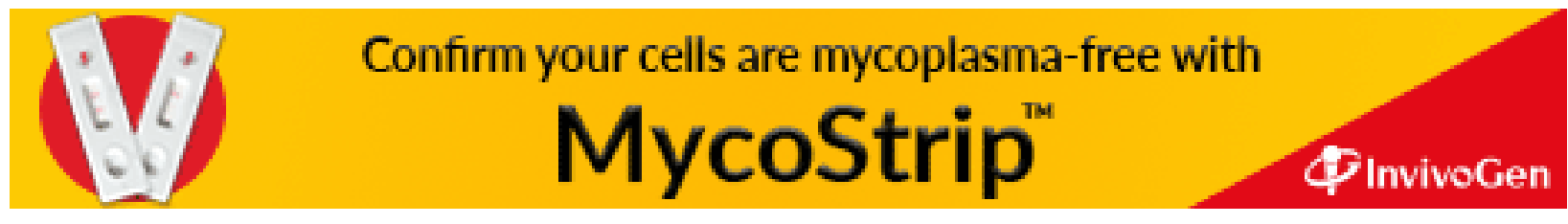

